\title{
Modelling exhaust plume mixing in the near field of an aircraft
}

\author{
F. Garnier, S. Brunet, L. Jacquin \\ Office National d'Etudes et de Recherches Aérospatiales, 8 rue des Vertugadins, 92190 Meudon, France
}

Received: 22 January 1997 / Revised: 16 April 1997 / Accepted: 6 May 1997

\begin{abstract}
A simplified approach has been applied to analyse the mixing and entrainment processes of the engine exhaust through their interaction with the vortex wake of an aircraft. Our investigation is focused on the near field, extending from the exit nozzle until about $30 \mathrm{~s}$ after the wake is generated, in the vortex phase. This study was performed by using an integral model and a numerical simulation for two large civil aircraft: a twoengine Airbus 330 and a four-engine Boeing 747. The influence of the wing-tip vortices on the dilution ratio (defined as a tracer concentration) shown. The mixing process is also affected by the buoyancy effect, but only after the jet regime, when the trapping in the vortex core has occurred. In the early wake, the engine jet location (i.e. inboard or outboard engine jet) has an important influence on the mixing rate. The plume streamlines inside the vortices are subject to distortion and stretching, and the role of the descent of the vortices on the maximum tracer concentration is discussed. Qualitative comparison with contrail photograph shows similar features. Finally, tracer concentration of inboard engine centreline of B-747 are compared with other theoretical analyses and measured data.
\end{abstract}

\section{Introduction}

The interaction of engine exhausts with the trailing vortices of an aircraft is an important topic for aviation impact on the atmosphere. Aircraft exhaust contains products resulting from combustion, usually designated as major species $\left(\mathrm{CO}_{2}, \mathrm{CO}, \mathrm{H}_{2} \mathrm{O}, \mathrm{NOx}\right)$, associated with a number of minor species (like $\mathrm{H}_{2} \mathrm{SO}_{4}, \mathrm{HNO}_{3} \ldots$ ), in gas or solid phase. Several recent studies (Brown et al.,

Correspondence to: F. Garnier e-mail: fgarnier@onera.fr
1996; Taleb et al., 1996) have shown the important part played by the system water vapour-sulphuric acid on aerosol formation in the wake.

For favourable ambient relative humidity and temperature, these emissions can lead to ice nucleation and growth processes that will form contrails in the atmosphere. These contrails may have an impact on cloudiness and may modify the Earth's radiative budget balance (Fortuin et al., 1995; Ponater et al., 1996).

However, the origin of contrails remains unclear, even though this problem has received much attention for many years (Appleman, 1953; Scorer and Davenport, 1970; Maxworthy, 1975; Schumann, 1996). One reason is the lack of knowledge of accurate partial vapour pressures of different species (mainly water and sulphuric acid) in the wake vortex. So, to assess these local variables which control the dispersion and dilution of the aircraft emissions, we have had to investigate the mixing process throughout the wake. The effect of trailing vortices on the mixing and chemistry of the jet engine has been studied by several authors (Miake-Lye et al., 1993; Garnier et al., 1997). In this study we focus on a simplified analysis of the mixing and dispersion of the engine jet in the near field of an aircraft.

In the present study, we use an integral model and a two-dimensional direct simulation of convection-diffusion equation (2D-DNS) to investigate the evolution of the mixing along the wake of a typical large transport aircraft, extending from the exit nozzle of the engine to the beginning of the vortex phase. Attention is concentrated on the evaluation of the surrounding mass flow (i.e. the vortical flow) which is radially drawn towards the jet engine across its conical surface.

The main physical processes in the near field of an aircraft wake are briefly presented in Sect. 2. Section 3 is devoted to the model's description and theoretical analysis. In Sect. 4 the initial conditions are described. Section 5 discusses the main results of the entrainment and mixing processes (characterised by the dilution ratio) of the effluents along the plumes of two large aircraft: a two-engine A-330 and a four-engine B-747, in 
cruise conditions. Section 6 draws conclusions and out looks.

\section{The nearfield interaction}

The wake of an aircraft is composed of two counterrotating wing-tip vortices. The vortical motion is generated by strong radial pressure gradients. As shown by several studies (Denison, 1995; Jacquin and Garnier, 1996; Gerz and Kärcher, 1996), for a large transport aircraft, the engine jets are initially virtually insensitive to the details of the vortex flow and later the exhaust emissions are captured by the vortices. At the early stage of vortex formation, the axial velocities are appreciable in the core of the vortices. But the details of this flow are not well known. However, the axial velocities in the core of the vortices decay more rapidly downstream than the tangential velocities (Ragab and Sreedhar, 1995). So, at least as a first approximation, in the jet phase, threedimensional (3-D) vortices evolve with time to twodimensional (2-D) vortices (Govindaraju and Saffman, 1971).

For modelling purposes, it is useful to define three regions in the entrainment and mixing process: a nearfield jet regime, a deflection regime and a shearing regime. They describe the jet evolution over the time periods of approximately, $0-1 \mathrm{~s}, 1-5 \mathrm{~s}, 5-30 \mathrm{~s}$ after the release of the hot exhaust from the nozzle exit into the atmosphere.

The nearfield jet regime is characterised by physical processes of usually co-flowing jets. For example, by means of a simplified computational model, Kärcher (1994) studied the expansion and cooling of a single engine jet of a subsonic B 747 .

The deflection regime corresponds to the entrainment of the jet engine towards the vortex core. From the studies on jets in crossflow (Rodi, 1982; Jacquin, 1994), it is assumed that the two basic parameters are the specific momentum flux of the jets (for plumes there is an analogous quantity, the specific buoyancy flux) and the vortex flow velocity. So the exhaust jet flow is subject to a balance of a centripetal force induced by the vortical motion and an inertial force induced by the specific momentum flux at the exhaust. The buoyancy body force is generated by the non-uniform density (i.e. temperature difference).

In the shearing regime, the shape of the plume is submitted to the shearing processes. This mechanism takes place as soon as the rotational component of the velocity varies across the plume surface. This occurs if the jet is sufficiently close to the vortex core, after the deflection regime. At this stage, the plume is subject to a centripetal buoyancy force. This corresponds to the buoyancy regime described by Miake-lye et al. (1993). However Lewellen and Lewellen (1996) using a large eddy simulation, have included a centrifugal buoyancy term, similar to the expression defined in the Boussinesq assumption. In the wake decay, the authors noted that this effect has little influence on the trapping of warm plume exhaust into the vortex core.

\section{The numerical models}

\subsection{Integral model}

An integral model has been developed to investigate continuously the jet mixing during the first two regimes described, i.e. up to its entrainment into the vortex core. This approach is described in a Lagrangian framework and uses the control volume concept to integrate the conservation laws of mass, momentum and energy.

Let us consider a jet element of diameter $\Delta$, crosssectional area $A=\pi \Delta^{2} / 4$ and mass $m=\rho A$ in the curvilinear frame $(\vec{s}, \vec{n}, \vec{b})$ (see coordinate system in Fig. 1). The jet is developing in the velocity field $\vec{V}\left(\hat{V}_{s}, \hat{V}_{n}, \hat{V}_{b}\right)$ induced by the superposition of two Gaussian vortices. It has been shown that the engine jets have a weak influence on global dynamics of the wake (Jacquin and Garnier, 1996). This approach is generalised to account for the transversal shear generated by the vortical motion.

The fundamental laws of dynamics is projected on the curvilinear frame $(\vec{s}, \vec{n}, \vec{b})$, by using the following geometrical relationships:

$\left\{\begin{array}{l}\vec{s}=\cos \gamma \cos \beta \vec{e}_{x}+\sin \gamma \vec{e}_{r}-\cos \gamma \sin \beta \vec{e}_{\theta} \\ \vec{n}=-\sin \gamma \cos \beta \vec{e}_{x}+\cos \gamma \vec{e}_{r}+\sin \gamma \sin \beta \vec{e}_{\theta} \\ \vec{b}=\sin \beta \vec{e}_{x}+\cos \beta \vec{e}_{\theta}\end{array}\right.$

This leads to:

$$
\left\{\begin{aligned}
\frac{\mathrm{d}}{\mathrm{d} s}\left(\rho A V_{s}\right) & =E \\
\frac{\mathrm{d}}{\mathrm{d} s}\left(\rho A V_{s}^{2}\right)= & E \hat{V}_{s}-A \frac{\partial \hat{p}}{\partial r} \sin \gamma+\frac{A}{r} \frac{\partial \hat{p}}{\partial \theta} \cos \gamma \sin \beta \\
\rho A V_{s}^{2} \frac{\mathrm{d} \gamma}{\mathrm{d} s}= & E \hat{V}_{n}-A \frac{\partial \hat{p}}{\partial r} \cos \gamma-\frac{A}{r} \frac{\partial \hat{p}}{\partial \theta} \sin \gamma \sin \beta \\
& +\rho A \frac{V_{s}^{2}}{r} \cos \gamma \sin ^{2} \beta+(\hat{\rho}-\rho) A g \sin \theta \\
-\rho A V_{s}^{2} \cos \gamma \frac{\mathrm{d} \beta}{\mathrm{d} s}= & E \hat{V}_{b}-\frac{A}{r} \frac{\partial \hat{p}}{\partial \theta} \cos \beta \\
& -\rho A \frac{V_{s}^{2}}{r} \cos \gamma \sin \gamma \sin \beta \\
& +(\hat{\rho}-\rho) A g \cos \theta
\end{aligned}\right.
$$

where subscripts $s, n$ and $b$ denote respectively the components tangential, normal and binormal to the jet path (see Fig. 1). $\hat{p}$ represents the external pressure field. External flow variables are denoted by a hat. The details of the basic equations are given in Jacquin and Garnier (1996).

Change in the mean concentration of a tracer in a jet is intimately related to the rate of entrainment, i.e. the rate at which ambient fluid is included within the jet boundaries. In the model, the turbulent diffusion and its effect on the expansion and cooling of the jet is based on the Morton-Taylor-Turner analysis and are accounted for by a single function $E$. The expression of this entrainment function writes: 


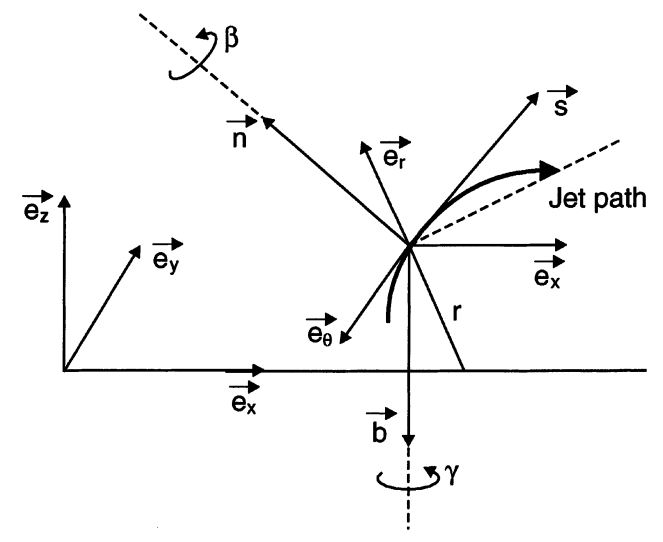

Fig. 1. Jet/wake interaction model: curvilinear frame

$$
E=\hat{\rho}\left[\alpha_{s}\left(V_{s}-\hat{V}_{s}\right)+\alpha_{n} \sqrt{\hat{V}_{n}^{2}+\hat{V}_{b}^{2}}\right] \Delta
$$

$\alpha_{s}$ and $\alpha_{n}$ are two empirical constants, obtained from experimental results and presented in Sect. 5.

Note that, since the present investigation is focused on the determination of the normalised plume concentration (defined as the dilution ratio) and temperature fields, it is performed using the energy conservation under the form of total enthalpy $H$ :

$\frac{\mathrm{d}\left(\rho A V_{s} H\right)}{\mathrm{d} s}=E \hat{H}$

The total enthalpy $H$ for the jet can be written:

$H=C_{p} T+\frac{V_{s}^{2}}{2}$

where $C_{p}$ represents the specific heat at constant pressure and has a constant value of $1005 \mathrm{~J} /(\mathrm{kgK})$ in the wake. $T$ is the jet temperature.

\subsection{The convection-diffusion equation}

For the shearing regime, the transport and mixing of the plume has been studied by means of the convectiondiffusion equation in an Eulerian framework.

The flow field examined here consists of a pair of idealised viscous core vortices which are suddenly turned on. The roll-up of the vortex sheet behind the wing into two counter-rotating cores is not taken into account. The kinematic condition (i.e. the linear addition of the two flow fields) is not quite exact, because the solution of the Navier-Stokes equations for a single viscous core vortex does not presume the presence of a second one. However it is a reasonable approximation even if the viscous flow fields do not satisfy Laplace's equation, because the distance between centres is large compared to the viscous core radius.

The velocity field is then represented by the superposition of two Lamb-Oseen vortices, with a descent (translation) velocity of $\frac{\Gamma}{2 \pi d}$, where $d$ denotes the separation between the two trailing vortices and $\Gamma$ the vortex circulation. By assuming the load distribution on the wing to be elliptic, $d$ is given by: $d=\frac{\pi}{4} b$, where $b$ denotes the wing span.

The tangential velocity corresponding to a superposition of two viscous core vortices is given by the following expression:

$$
\begin{aligned}
& \overrightarrow{\hat{V}}_{\theta}\left(r, r^{\prime}, t\right) \\
& =\frac{\Gamma}{2 \pi} \times\left\{\frac{1-\exp \left(-r^{2} / 4 v t\right)}{r} \vec{e}_{\theta}-\frac{1-\exp \left(-r^{\prime 2} / 4 v t\right)}{r^{\prime}} \vec{e}_{\theta}{ }^{\prime}\right\}
\end{aligned}
$$

where $r$ and $r^{\prime}$ represent the separation distances between the considered points and the two vortex centres (see Fig. 2) and $v$ is the viscosity. The vortex centres are placed symmetrically with respect to the vertical axis and move in the negative vertical direction.

For simplicity and to introduce a general approach, the flow field is considered here as a binary mixture. Thus a two-stream problem is examined, having uniform properties over one part of the flow field which is called the hot plume stream, and different uniform properties over the rest of the flow field, called the atmospheric flow stream. Furthermore one assumes that there are no exhaust products in the ambient air stream and no ambient air in the plume stream.

Based on analysis of mixing, the following assumptions are applied:

1. The mass diffusion velocities of species are expressed in terms of Fick's law.

2. Thermal diffusion of emissions is neglected.

3. The diffusion coefficients $D_{i}$ of the different species with respect to the mixture are constant and equal $\left(D_{i}=D\right.$ for all the species of the hot plume and the external flow).

4. Specific heats at constant pressure for all species take the same value $C_{p}$.

5. In many calculations concerning gases the Lewis number, Le, is taken to be equal to one. The Lewis number has a real measureable value for a given fluid (often close to one for gases) and is only taken to be an approximate value for particular purposes. This approximation is frequently helpful in theoritical mixing and combustion analyses (Williams, 1985). Le can be defined from the Prandtl number, Pr, and the Schmidt

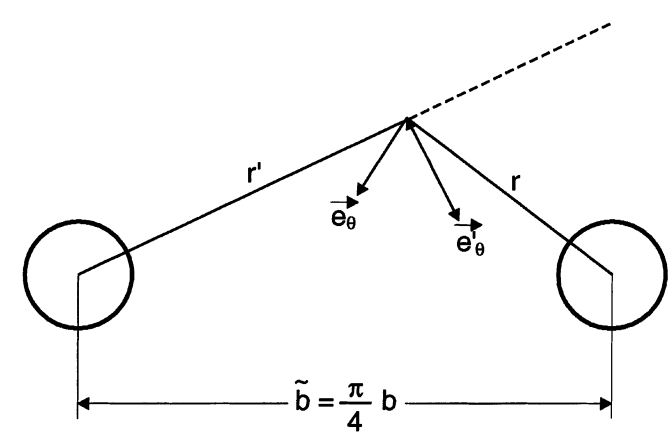

Fig. 2. Wake vortex as a superposition of two vortices: notations 
number, Sc: $\mathrm{Le}=\mathrm{Sc} / \mathrm{Pr}$. In this analysis, we will take $\operatorname{Pr}=\mathrm{Sc}=1$. As shown by Kärcher (1994) this assumption will be considered as representative.

For favourable ambient relative humidity and temperature, ice formation will have occurred and the latent heat release associated can modify the velocity field. This process is not included in the present study. Considerable simplification may be introduced if density variations associated to heat release are neglected. This constant density assumption decouples the velocity field from the mass fractions of species and temperature. Known solutions of incompressible Navier-Stokes equations may then be imposed, and the problem is reduced to solve the following species and energy balance equations.

$$
\begin{aligned}
& \frac{\partial}{\partial t}\left(\rho F_{a}\right)+\nabla \cdot\left(\rho V F_{a}\right)=\nabla \cdot\left(\rho D \nabla F_{a}\right) \\
& \frac{\partial}{\partial t}\left(\rho F_{j}\right)+\nabla \cdot\left(\rho V F_{j}\right)=\nabla \cdot\left(\rho D \nabla F_{j}\right) \\
& \frac{\partial}{\partial t}\left(\rho C_{p} T\right)+\nabla \cdot\left(\rho V C_{p} T\right)=\nabla \cdot(\lambda \nabla T)
\end{aligned}
$$

where $F_{a}$ and $F_{j}$ refer respectively to the mass fractions of the atmospheric species and the exhaust plume species. $\lambda$ is the thermal conductivity and $T$, the temperature field.

Following Williams (1985), it is convenient to introduce the dilution ratio $Z$ (or the mixture variable):

$Z=\frac{F_{j}-F_{a}+F_{a, 0}}{F_{j, 0}+F_{a, 0}}$

where $F_{j, 0}$ and $F_{a, 0}$ describe respectively the initial mass fraction of the exhaust species and atmospheric species. $Z$ is defined as a passive scalar which is initially assumed to have a value of one inside the plume and zero in the ambient air. Furthermore this conserved scalar does not influence the flow dynamics. This variable satisfies the following conservation equation:

$\rho \frac{\partial Z}{\partial t}+\rho V \cdot \nabla Z=\nabla \cdot(\rho D \nabla Z)$

where $D$ is the diffusivity of the scalar. Thus the mass fraction $F_{i}$ of a particular species $i$ at any point in the solution may be given explicitly from the variable $Z$ :

$F_{i}=\left(F_{i a, o}-F_{i a, o}\right) Z \quad i=1, \ldots, N$

and a similar expression is obtained directly for $F_{a}$ and $F_{j}$

$F_{a}=F_{a, 0}(1-Z)$

$F_{j}=F_{j, 0} Z$

If one assumes that the rate of change of pressure as function of time is negligible, the energy equation has the same form as the balance equation of species (see Eq. 7), the temperature profile can be expressed also in terms of $Z$, which would be obtained by the expression:

$T=T_{a, 0}+\left(T_{j, 0}-T_{a, 0}\right) Z$
Note that, from the present analysis, chemical reactions can be introduced without difficulties. The passive scalar field $Z$ is a scalar quantity that is neither created nor destroyed by chemical reactions. However the aim of this work is to study "frozen" temperature and concentration profiles which are obtained from pure mixing, without chemical reactions.

This simulation is performed for a laminar case, using a Reynolds number based on the circulation $\frac{\Gamma}{v}$ and equal to 3000 . So, the laminar vortex core grows only by viscous diffusion. At high Reynolds numbers (flight conditions) the turbulence must be considered but its precise role is unclear. Furthermore Zeman (1995) showed that the turbulence has a weak influence on the persistence and the growth of the vortices.

The aircraft wake problem is clearly formulated in an infinite domain. However to carry out the calculation, we have had to determine a finite field. A squared domain of size $4 \mathrm{~b}$ (where $\mathrm{b}$ represents the wing span) is considered. It is now convenient to introduce dimensionless variables. To outline the relative importance of the physical processes accounted for in Eq. (9), the dimensional variables are scaled with $\Gamma$, the circulation of the vortices and $d$ the separation distance between the two trailing vortices. They can then be written in a twodimensional form:

$\boldsymbol{X}=\mathrm{d} X^{*}, \quad \boldsymbol{Y}=\mathrm{d} Y^{*}, \boldsymbol{t}=\left(\mathrm{d}^{2} / \Gamma\right) t^{*}$, where the asterisk refers to dimensionless variables of order 1 .

Then the equation for $Z$ can be written in a dimensionless form and takes the following expression:

$$
\frac{\partial Z}{\partial t^{*}}+U^{*} \frac{\partial Z}{\partial X^{*}}+V^{*} \frac{\partial Z}{\partial Y^{*}}=\frac{1}{\operatorname{Re} S c}\left(\frac{\partial^{2} Z}{\partial X^{* 2}}+\frac{\partial^{2} Z}{\partial Y^{* 2}}\right)
$$

where $R e$ and $S c$ represent respectively the Reynolds and Schmidt number $(\mathrm{Sc}=v / D) . \quad U^{*}$ and $V^{*}$ are the cartesian velocity coordinates, determined from the tangential velocity in Eq. (6).

The dimensionless tangential velocity for one viscous core vortex takes the following form:

$$
V_{\theta}^{*}=\frac{1}{2 \pi r^{*}}\left[1-\exp \left(-\frac{\operatorname{Re} r^{* 2}}{4 t^{*}+R_{c}^{* 2}}\right)\right]
$$

where $r^{* 2}=X^{* 2}+Y^{* 2}$ and $R_{c}^{* 2}$ is the initial core radius given in the next section.

The relevant boundary conditions for $Z$ are $Z=0$ at $Y \rightarrow \pm \infty$ and at the lateral boundaries of the box: $\frac{\partial Z}{\partial X^{*}}=0$. If the size of the computational domain is large compared to the characteristic scale of the flow field, it is expected that the results are not dependent upon the lateral boundaries. But as the vortex flow grows as a function of time, the calculation must be stopped when the vortex approaches the limits of the domain.

The computations are performed on a fine-grid of $601 \times 601$ grid points in order to resolve the details of the plume structure. The numerical method adopted here is an implicit finite-difference scheme, developed by Peyret (1983). A flux corrected transport filter based on the diffusion-antidiffusion technique (Boris and Book, 1976) is added to avoid the numerical oscillations. 
A more complete description of this method is given in Laverdant and Candel (1988).

\section{Initial conditions}

The aerodynamic aircraft parameters and flight conditions are described in Table 1 for a two-engine civil transport aircraft: an Airbus A-330 and for a fourengine aircraft: a Boeing 747. The thermodynamic conditions at the exit of a typical engine are given at the end of the Table 1. The initial vortex core radius $R_{c}^{*}$ is taken arbitrarily to be constant, equal to $1 \mathrm{~m}$, only in the first stage of the integral calculation (i.e. from the exit nozzle up to the shearing regime). Other calculations with different vortex core radius have been carried out. In the near field this parameter has a weak influence on the mixing process (data not shown). The simulation with the integral model is stopped when the jet boundary encounters the vortex centre. There, the jet has become mostly dominated by the shearing process.

In this latter regime, we start by assuming that the tracer concentration and temperature fields are approximately Gaussian. From the average variables determined by the integral method, the Gaussian plume amplitude for the temperature field is calculated by the following expression:

$T_{g} \int_{-\infty}^{+\infty} \exp \left[-4\left(\frac{x}{\Delta}\right)^{2}\right] \mathrm{d} x=T_{m} \Delta$

where $T_{g}$ depicts the Gaussian plume amplitude for the temperature and $T_{m}$ represents the mean temperature calculated from the integral model. $\Delta$ is the plume diameter.

Table 1. Aircraft parameters for a two engine Airbus A-330 and a four engine Boeing B-747 studies and thermodynamic conditions at the exit of a typical engine.

\begin{tabular}{|c|c|c|}
\hline & $\begin{array}{l}\text { Two-engine } \\
\text { Airbus A-330 }\end{array}$ & $\begin{array}{l}\text { Four-engine } \\
\text { Boeing B-747 }\end{array}$ \\
\hline \multicolumn{3}{|l|}{ Flight conditions: } \\
\hline Altitude $(\mathrm{km})$ & 10 & 10 \\
\hline Ambient temperature (K) & 220 & 220 \\
\hline Ambient pressure $(\mathrm{hPa})$ & 240 & 240 \\
\hline Ambient density $\left(\mathrm{kgm}^{-3}\right)$ & 0.38 & 0.38 \\
\hline Aircraft weight $(\mathrm{N})$ & $2.0810^{6}$ & $2.8010^{6}$ \\
\hline Span b (m) & 60.3 & 60 \\
\hline Aspect ratio & 9.3 & 7.5 \\
\hline Wing surface $\left(\mathrm{m}^{2}\right)$ & 361.3 & 480 \\
\hline Circulation $\Gamma\left(\mathrm{m}^{2} \mathrm{~s}^{-1}\right)$ & 540 & 540 \\
\hline Lift coefficient & 0.5 & 0.42 \\
\hline Aircraft velocity $\left(\mathrm{ms}^{-1}\right)$ & 252 & 250 \\
\hline $\begin{array}{l}\text { Distance engine/vortex } \\
\text { centre }(\mathrm{m})\end{array}$ & 14 & 14 \\
\hline $\begin{array}{l}\text { Nozzle diameter primary } \\
\text { flux }(\mathrm{m})\end{array}$ & 0.61 & \\
\hline Jet exhaust temperature $(\mathrm{K})$ & 580 & \\
\hline Jet exhaust velocity $(\mathrm{m} / \mathrm{s})$ & 480.3 & \\
\hline
\end{tabular}

A similar expression can be written for the dilution ratio. We set the initial plume width so that $1 \%$ of effluent encounters the vortex centre (Fig. 9a).

So, when the direct numerical simulation runs, a plume Gaussian distribution of a scalar (dilution ratio and temperature) is introduced in the surrounding of two Lamb-Oseen vortices.

\section{Results and discussion}

The empirical constants for the integral model have been chosen in the following way: from experiments on coaxial gas jets (Forstall and Shapiro, 1950), the coefficient $\alpha_{s}$ is taken to be 0.15 . The value of $\alpha_{n}$ is close to one and is determined from experiments on the study of jets in a cross flow (Jacquin, 1994; Rodi, 1982). Figure 3 shows the evolution of normalised axial velocity versus axial distance $x / \Delta$, where $\Delta$ is the diameter of the nozzle exit. The results from the integral model (squares in Fig. 3) do not reproduce the core region well (i.e. up to 10 diameters behind the nozzle) where the velocity profile remains unchanged. The integral techniques provide the average jet properties and do not take into account the radial profile evolution. However a good agreement between integral results and experimental data (triangles in Fig. 3) is observed in the far field of the jet phase.

In this study the wing-tip vortices of the two aircraft: A-330 and B-747 are assumed to be fully rolled-up and the generation region is not taken into account by the modelling.

Figure 4 shows a typical large aircraft contrail observed from the ground a few seconds after the passage of the aircraft. The features of this observation depend strongly on the ambient relative humidity and temperature. If these values are low enough, in the early stage of the wake only the ice crystals formed by the aircraft exhaust trapped in the vortex core are seen. A qualitative comparison with the calculations of the wing-tip-vortex/jet exhaust interaction using the integral

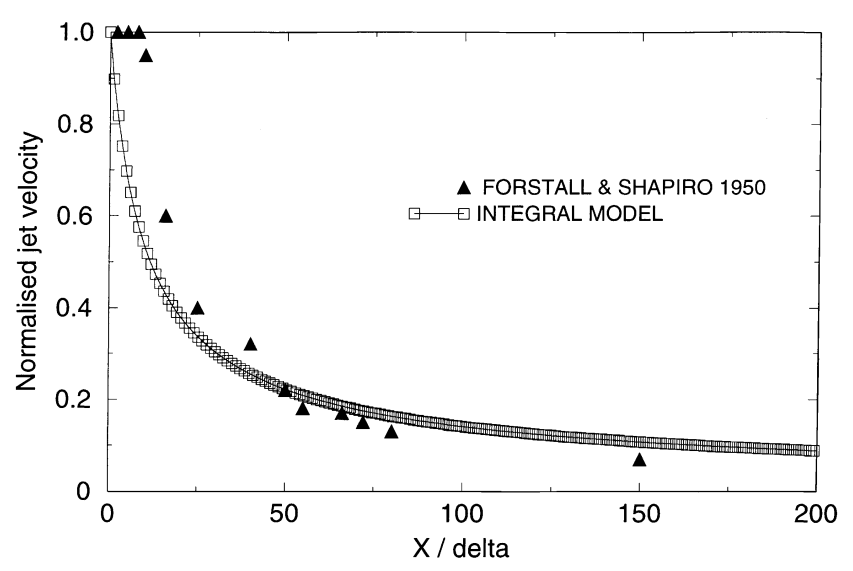

Fig. 3. Normalised velocity as a function of downstream distance $\mathrm{X} / \Delta$, where $\Delta$ is the exhaust jet diameter. Comparison with the Forstall and Shapiro (1950) experimental data 

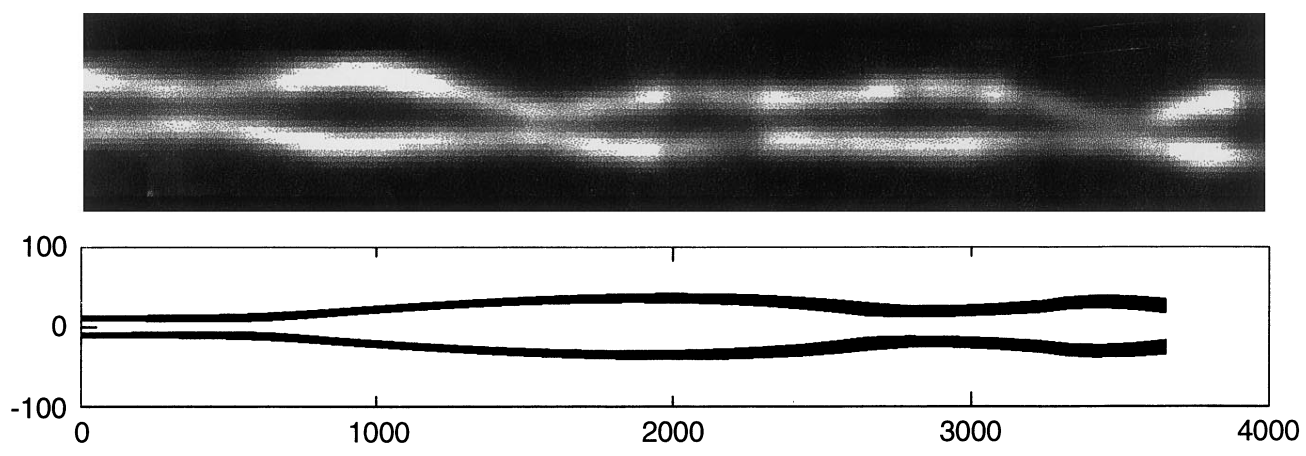

Fig. 4. Engine jet trajectory behind a typical large aircraft. This calculation is carried out using the integral model. Qualitative comparison with a contrail photograph method shows the deflection of the engine jet streamlines generated by the centripetal force.

Figure 5 depicts the effect of the body buoyancy force on the dilution ratio and mean temperature. These variables, are calculated with and without the buoyancy force. It is apparent that this force enhances the mixing but only after a distance of about two or three wing spans behind the aircraft. An increase of the difference between the two calculations, performed with and without the buoyancy force, is observed as a function of downstream distance. Then, the specific buoyancy flux becomes large enough to balance the jet specific momentum flux, when the trapping in the vortex core has occurred. It corresponds to the end of the co-flowing jet regime defined in Sect. 2.

Figure 6 shows the results obtained when considering an isolated jet flow (i.e. without the action of the wingtip vortices) and an interaction with a single vortex and
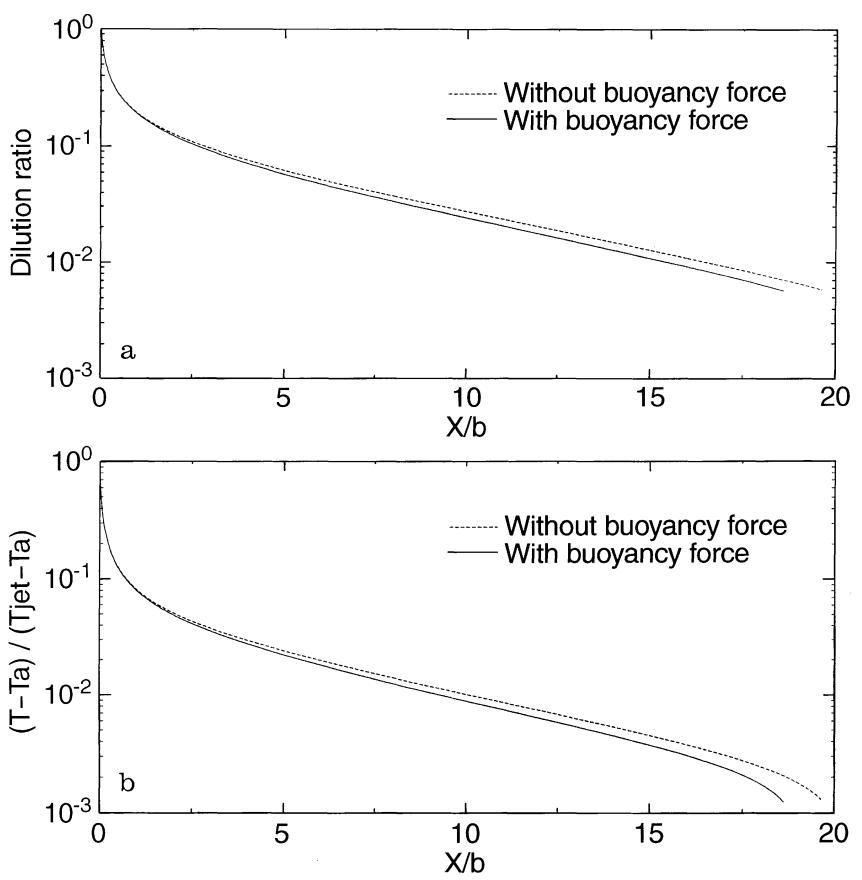

Fig. 5. Effect of the buoyancy force on a the dilution ratio and b normalised mean temperature, plotted versus $\mathrm{x} / \mathrm{b}$ ( $\mathrm{b}$ being the span) for a twin-engine Airbus 330. This calculation is carried out using the integral model (Tjet is the jet temperature at the exit nozzle plan and Ta the ambient temperature) with the two wing-tip vortices. Comparison shows that the action of the counter rotating system increase the mixing and entrainment processes and make the deflection and trapping of exhaust jet more efficient than in a single vortex. As a consequence, at 18 wing spans (about one kilometre behind the aircraft), the dilution factor is five times smaller than the dilution factor calculated without the effect of the vortices (i.e. with an axisymmetric co-flow jet assumption), and about three times smaller than that determined from a single vortex assumption. Finally, these results illustrate the importance of including the jet-vortex interaction when determining the mixing phenomena.

From Fig. 7 the influence of the engine location on the mixing rate can be seen. This calculation is performed for an inboard and outboard engine jet on a B-747. The results show the importance of the position of the engine jet with respect to the wing-tip, on the dilution calculation. For the outboard engine, closest to the wingtip, the dilution ratio decreases very rapidly. At a distance of about two wing spans behind the aircraft the outboard jet is completely trapped by the counter rotating system and in this case the integral calculation, which simulates only the deflection regime, is stopped. By then the hot plume has become dominated by the shearing process. For the inboard jet, the mixing and entrainment is less efficient, thus the dilution factor is much greater (by a factor of 2.6 at two wing spans). It is due to the relative distance from the wing-tip. The integral calculation runs for a longer distance behind the B-747. The trapping of the inboard jet inside the vortex core is less effective than that of the outboard engine jet.

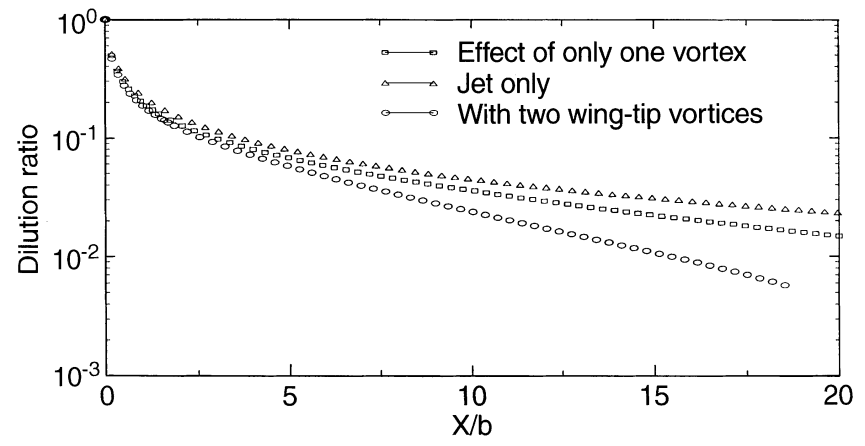

Fig. 6. Effect of the wing-tip vortices on the dilution ratio of a twinengine A-330, obtained using integral calculation 


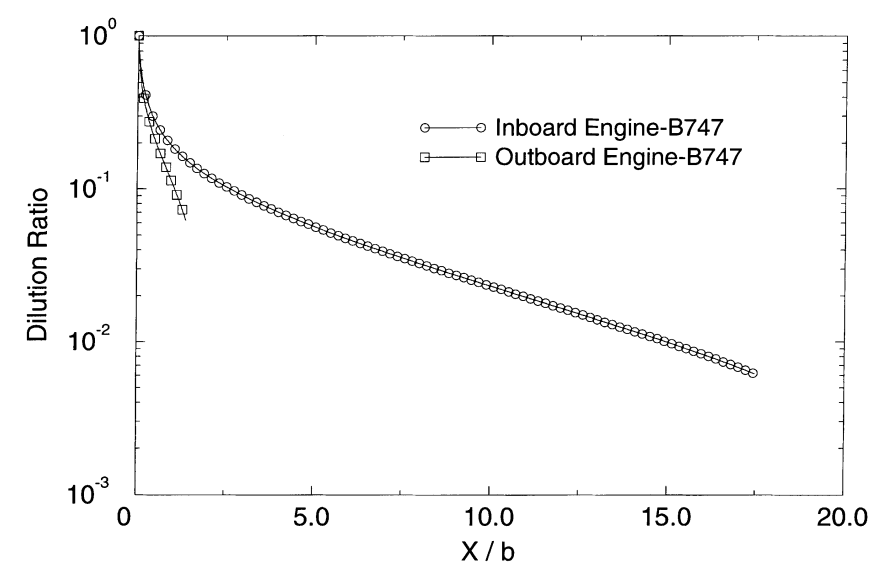

Fig. 7. Dilution ratio profiles comparing the inboard and outboard engine jet results for a four-engine Boeing 747. Calculated from integral model

Figures 8 and 9 show the results given by the numerical simulation of a convection-diffusion equation. The dilution ratio (considered as a tracer concentration) is calculated at different locations inside the wake vortex. The maximum tracer concentration (squares in Fig. 8) follows the exhaust hot plume throughout the wake. After $20 \mathrm{~s}$ of plume production, the dilution ratio is of the order of $5 \cdot 10^{-4}$. The increase of the dilution ratio calculated on the vortex axis, shows the rapid distortion and entrainment of the exhaust emissions towards the vortex core (triangles in Fig. 8). Figure 9a-d shows the evolution with time of the crossflow distribution of dilution ratio field. The drawing of the aircraft is shown for scale and does not represent the location relative to the wake. The entrainment and mixing processes in this case show a stretching and a distortion of the interface between plume and ambient air (Fig. $9 b$ ), followed by a relatively large-scale engulfment into the vortex core (Fig. 9c, d). The vortices descend under

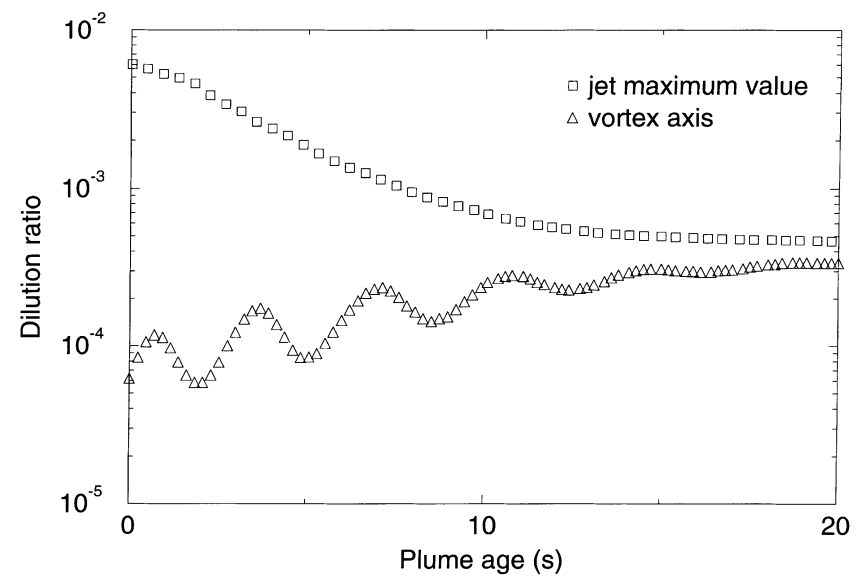

Fig. 8. Dilution ratio profiles calculated at different locations inside the wake vortex of a twin-engine A-330. These results are computed from a direct numerical simulation of a convection-diffusion equation. The squares denote the maximum of tracer concentration. The triangles represent the computation of the dilution ratio on the vortex axis mutual interaction and the hot plume is trapped around the vortex core. This is also observed in Fig. 8 where the plot of maximum dilution ratio (squares in Fig. 8) is located on the vortex line (triangles in Fig. 8). This is the beginning of the detrainment process and the mixing is then driven by the descent velocity. The characteristic time scale for the trailing vortices to descent by mutual induction to a distance $d$ (the vortex pair separation) is defined by: $T_{l}=2 \pi \frac{d^{2}}{\Gamma}$. With the aircraft parameters given in Table $1, T_{l}$ is of the order of $26 \mathrm{~s}$. It corresponds to the life-time of the aircraft wake. Then the break-up process can appear, generated by Crow sinusoidal instability. Atmospheric turbulence and stratification enter in the stability of the wake vortices in the form of non-linear forcing term (Crow and Bate, 1975), and as a consequence the two-dimensional representation of the flow field is not valid any more. The far field phenomena can be only characterised by a three-dimensional large eddy simulation. The present work is obviously limited to the near field.

Figure 10 shows the evolution of the tracer concentration (analogous to the dilution ratio) of the inboard engine jet for the Boeing 747, as a function of aircraft wake age. A comparison of three different analyses with measured data (Schumann et al., 1995) has been plotted on this figure. The UNIWAKE analysis (Anderson et al., 1996), based on the average turbulent Navier-Stokes equations seems to underpredict the mixing rate, compared to the Schumann data. At the plume age $T_{l}$ described already, the maximum tracer concentration remains constant. The Lewellen and Lewellen (1996) analysis, based on the unsteady Navier-Stokes equations (large eddy simulation), captures the wake breakup phenomena. Then the tracer concentration decreases again and is consistent with the Schumann data (at least after extrapolation). The results calculated from the present and the two previous analyses are of the same order of magnitude. Furthermore, a comparison with another large eddy simulation analysis carried out by Gerz and Kärcher (1996) is also in close agreement in the early stage of the wake.

The best agreement is found in the shearing regime (From $5 \mathrm{~s}$ to about $20 \mathrm{~s}$ of plume age) which is analysed by a laminar approach. This seems to confirm Zeman's (1995) assumption on the laminar growth of the trailing vortices. However, even if the extrapolation of these results compared to the Schumann data seems to be acceptable, our approach does not take into account the break-up process. The decreasing of the tracer concentration is only due to the descent of the vortices.

\section{Conclusion}

We have used an integral model and a direct numerical simulation of a passive tracer field to investigate the entrainment and mixing between the hot exhaust jet and the surrounding air. The objective of this simplified approach is to provide a computational model at low cost (compared to the large eddy simulation), to be able to run on a work station. 


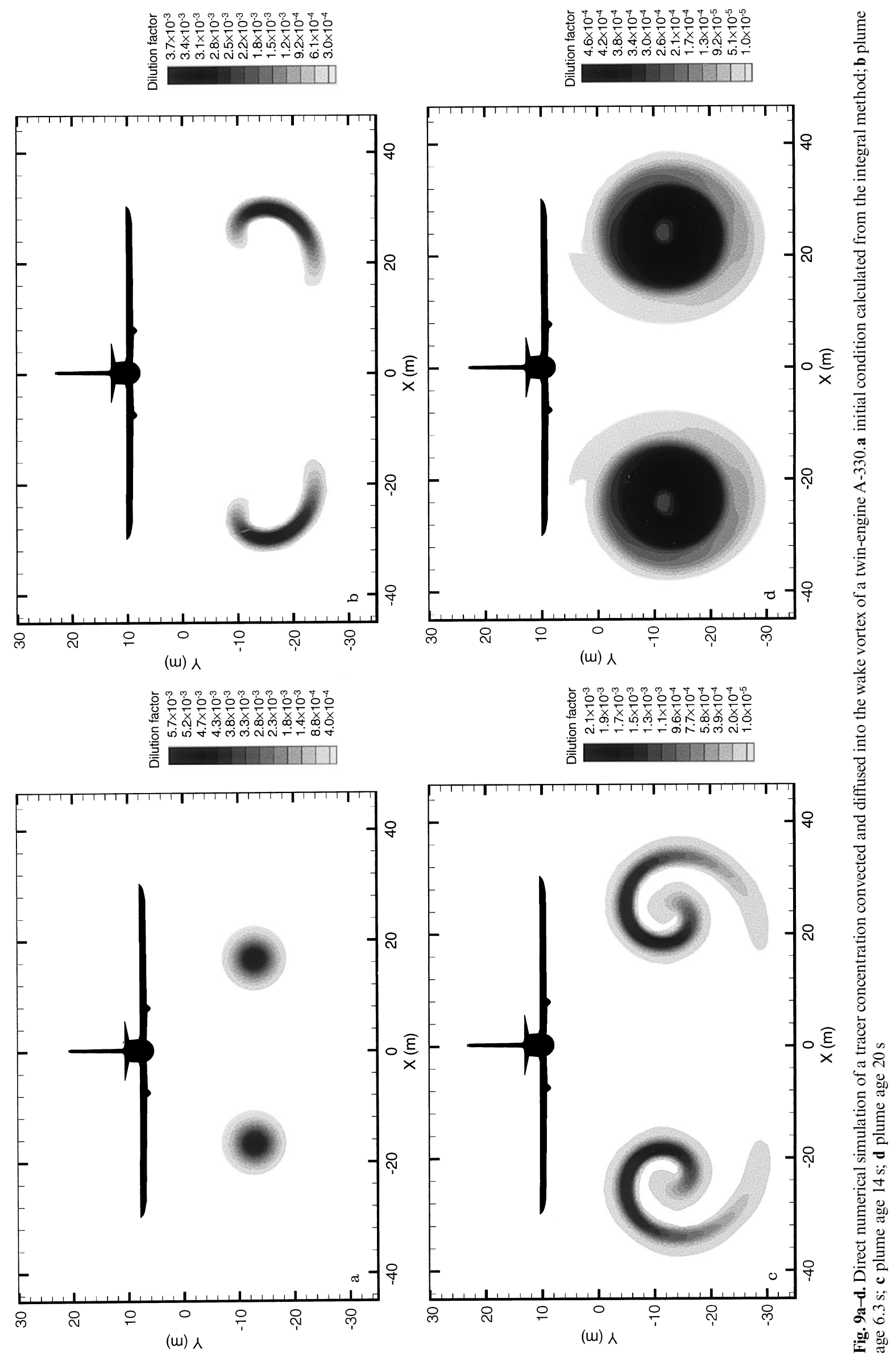




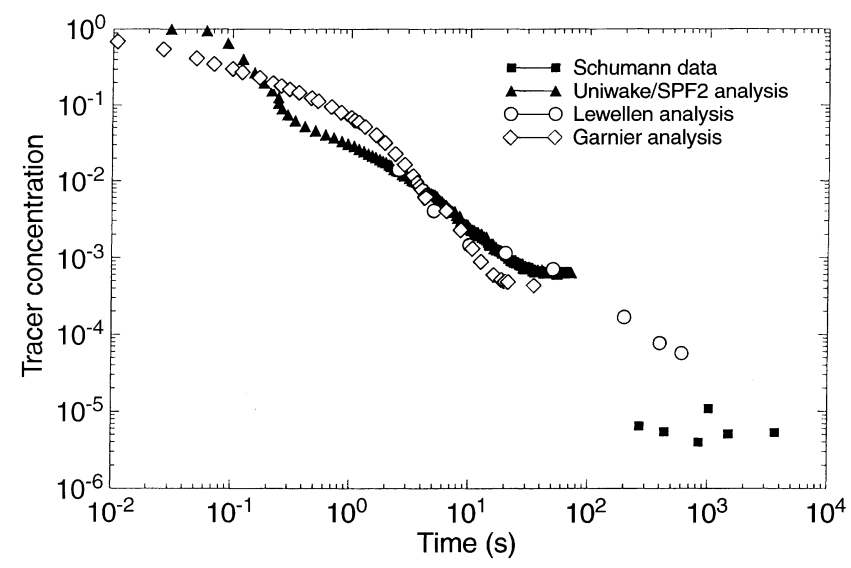

Fig. 10. Axial tracer concentration plots of inboard engine centreline of B-747. Comparison of three theoretical analyses with the Schumann data: UNIWAKE analysis, Lewellen analysis and the present approach

This analysis is performed in the near field of the wake vortex of two large transport aircraft: a twinengine Airbus 330 and a four-engine Boeing 747, up to about $30 \mathrm{~s}$ after the wake is generated. It is expected that the mixing process in the early stage of the wake could affect the microphysical properties of the contrails at a later time.

The wing-tip vortices contribute largely to enhance the mixing and entrainment processes. The engine jet location has an important influence on the mixing rate. But quantification of these effects remains very difficult, mainly due to the lack of experimental data.

The mixing is modified by the deflection, the distortion and the stretching of the plume, produced by the transversal shear inside the vortex core. The dilution results characterised only by physical processes of usual co-flowing jets are very different (i.e. about six times greater) from those accounting for the interaction with the wing-tip vortices.

The calculations of the dilution ratio have been compared with the results given by more complete analyses: an averaged Navier-Stokes model (Anderson et al., 1996) and a large eddy simulation (Lewellen and Lewellen, 1996). Comparison shows that the models are in good agreement. The good quality of the results seems to show that the turbulence in the beginning of the vortex regime (from $4 \mathrm{~s}$ to approximately $20 \mathrm{~s}$ ) is not the key parameter in the entrainment and mixing phenomena.

However, our work is obviously limited to the near field regime. Atmospheric effects (stratification, turbulence, shear) are not included in this study but dominate the dynamics and the mixing of the aircraft exhaust in the far field.

Acknowledgements. The authors would like to thank Dr. Alain Laverdant for providing a part of the model code and advice for this work. Furthermore, we want to thank the referees who helped us to correct the manuscript.

Topical Editor L. Eymard thanks K. Gierens and R. C. MiakeLye for their help in evaluating this paper.

\section{References}

Anderson, M. R., R. C. Miake-Lye, R. C. Brown, and C. E. Kolb, Numerical modeling of reacting engine plumes and aircraft wake interactions, AIAA Paper, 96-0948, 1996.

Appleman, H., The formation of exhaust condensation trails by jet aircraft, Bull. Am. Meteorol. Soc., 34, 14-20, 1953.

Boris, J. P., and D. L. Book, Flux corrected transport III- minimal error FCT algorithms, J. Comp. Phys., 20, 397-432, 1976.

Brown, R.C., R. C. Miake-Lye, M.R. Anderson, C.E. Kolb, and T. J. Resh, Aerosol dynamics in near field aircraft plumes, J. Geophys. Res., in press, 1996.

Crow, S. C., and E.R. Bate, Lifespan of trailing vortices in a turbulent atmosphere, J. Aircraft, 13, 476-482, 1975.

Denison, M. R., Overview of high-altitude aircraft emissions models. AIAA Paper, 95-3940, 1995.

Forstall, W.JR. and A.H. Shapiro, Momentum and mass transfer in coaxial gas jets, J. Appl. Mech., 399-408, 1950.

Fortuin, J. P. F., R. Van Dorland, W. M. F. Wauben, and H. Kelder, Greenhouse effects of aircraft emissions as calculated by a radiative transfert model, Ann. Geophysicae, 13, 413-418, 1995.

Gerz, T., and B. Kärcher, Dilution of aircraft exhaust and entrainment rates for trajectory box models, in Impact of Aircraft Emissions upon the Atmosphere. Paris, October 15-18, 1996.

Garnier, F., C., Baudoin, P. Woods, and N. Louisnard, Engine emission alteration in the near field of an aircraft, Atma. Environ. 31, 1767-1781, 1997.

Govindaraju, S. P., and P. G. Saffman, Flow in a turbulent vortex, Phys. Fluids, 14, 2074-2080, 1971.

Jacquin, L., Phenomenological description and simplified modeling of the vortex wake issuing from a jet in a crossflow, La Rech. Aéro., 2, 117-133, 1994.

Jacquin, L., and F. Garnier, On the dynamics of engine jets behind a transport aircraft, in The Characterization and Modification of Wakes from Lifting Vehicles in Fluids, AGARD CP 584, 37.137.8, May 1996.

Kärcher, B., Transport of exhaust products in the near trail of a jet engine under atmospheric conditions, J. Geophys. Res., 99, 14509-14517, 1994.

Laverdant, A., and S. Candel, A numerical analysis of a diffusion flame-vortex interaction, Combust. Sc. Techol. 60, 79-96, 1988.

Lewellen, D. C., and W. S. Lewellen, Large-eddy simulations of the vortex-pair breakup in aircrfaft wakes, AIAA J. 34, 11, 23372345, 1996.

Maxworthy, T., The motion of aircraft trailing vortices, J. Appl. Mech., 42, 2, 279-282, 1975.

Miake-Lye, R. C., M. Martinez-Sanchez, R. C. Brown, and C. E. Kolb, Plume and wake dynamics, mixing, and chemistry behind a high speed civil transport aircraft, J. Aircraft, 30, 467-479, 1993.

Peyret, R., and T. D. Taylor, Computational methods for fluid flow. Springer-Verlag, New York, Berlin Heidelberg, 68, 1983.

Ponater, M., S. Brinkop, R. Sausen, and U. Schumann, Simulating the global atmospheric reponse to aircraft water vapour emissions and contrails: a first approach using a GCM, Ann. Geophysica,14, 941-960, 1996.

Ragab, S., and M. Sreedhar, Numerical simulation of vortices with axial velocity deficits, Phys. Fluids, 7, 549-558, 1995.

Rodi, W., Turbulent buoyant jets and plumes, Pergamon, Oxford, 1982.

Schumann, U., P. Konopka, R. Baumann, R. Busen, T. Gerz, H. Schlager, P. Schulte, and H. Volkert, Estimate of diffusion parameters of aircraft exhaust plumes near the tropopause from nitric oxyde and turbulence measurements, J. Geophys. Res., 100, 14147-14161, 1995.

Schumann, U., Contrail formation from aircraft exhausts, Meteorol. Z., N.F. 5, 4-23, 1996.

Scorer, R. S., and L. J. Davenport, Contrails and aircraft downwash, J. Fluid Mech., 43, 451-464, 1970. 
Taleb, D., R. McGraw, and P. Mirabel, Microphysical study of aerosol formation in the wake of an aircraft, J. Geophys. Res., in press, 1996.

Williams, F. A., Combustion Theory. 2nd edn., Benjamin Cummings, CA., 1985.
Zeman, O., The persistence of trailing vortices: a modeling study, Phys. Fluids, 7, 135-143, 1995. 